\title{
Optical and Infrared Observations of Diffuse Clouds
}

\author{
Benjamin J. McCall \\ Departments of Chemistry and Astronomy, University of Illinois at Urbana-Champaign, \\ Urbana, IL 61801, USA \\ email: bjmccall@uiuc.edu
}

\begin{abstract}
In the past several years, great progress has been made on the spectroscopy of polyatomic molecules in diffuse interstellar clouds. In this talk, I will review recent developments involving $\mathrm{H}_{3}^{+}, \mathrm{C}_{3}$, and the Diffuse Interstellar Bands (DIBs).

The simplest polyatomic molecular ion, $\mathrm{H}_{3}^{+}$, has long been recognized as the cornerstone of ion-neutral chemistry in dense molecular clouds (Herbst \& Klemperer 1973; Watson 1973). However, in diffuse clouds (where electrons are abundantly produced from photoionization of atomic carbon) the $\mathrm{H}_{3}^{+}$number density was expected to be considerably lower than in dense clouds, owing to the efficiency of electron recombination. It was, therefore, a surprise when a large column density of $\mathrm{H}_{3}^{+}$was detected (McCall et al. 1998) in the diffuse line of sight towards Cygnus OB2 12, and subsequently in a sample of heavily reddened diffuse sightlines (McCall et al. 2002). Recently, we have detected $\mathrm{H}_{3}^{+}$even in the classical diffuse cloud sightline towards $\zeta$ Persei; together with a new measurement of the electron recombination rate coefficient, this result suggests that the cosmic-ray ionization rate is much higher in diffuse clouds than in dense clouds (McCall et al. 2003a)!

In 2001, interstellar $\mathrm{C}_{3}$ was first detected by J. P. Maier and colleagues (Maier et al. 2001) in three diffuse cloud sightlines. This was quickly followed up by another detection (Roueff et al. 2002) and a survey conducted at low-resolution (Oka et al. 2003). This was followed by a high-resolution survey (Ádámkovics, Blake, \& McCall 2003) that yielded rotationally resolved spectra of $\mathrm{C}_{3}$ in 10 sightlines. Much like $\mathrm{C}_{2}, \mathrm{C}_{3}$ has no permanent dipole moment, and therefore its rotational distribution serves as a sensitive diagnostic of both temperature and density.

The existence of larger polyatomic molecules in diffuse clouds is clear from the presence of the DIBs, which have remained an enigma since their discovery some eight decades ago. A recent survey of the DIBs at the Apache Point Observatory has resulted in a uniform sample of DIB spectra with much higher signal-to-noise ratios than previously available. Some early results from the analysis of the survey data include the identification of a set of DIBs that are related to $\mathrm{C}_{2}$ (Thorburn et al. 2003) as well as some well-correlated pairs of DIBs. I will discuss our recent results.
\end{abstract}

\section{Introduction}

The first molecules to be detected in the interstellar medium $\left(\mathrm{CH}, \mathrm{CH}^{+}\right.$, and $\left.\mathrm{CN}\right)$ were found using optical spectroscopy in what we now refer to as diffuse clouds. Polyatomic molecules, such as $\mathrm{NH}_{3}, \mathrm{H}_{2} \mathrm{O}$, and $\mathrm{H}_{2} \mathrm{CO}$, were then detected by radioastronomers in dense molecular clouds, and much of the work in the young field of astrochemistry has been focused on these clouds and the star-forming regions within them.

However, in recent years many polyatomic molecules have been detected in diffuse clouds, both at optical and infrared wavelengths (as discussed in this contribution) and at radio wavelengths (as discussed by Liszt, this volume). This is somewhat surprising, considering that diffuse clouds have lower number densities, higher radiation fields, and 


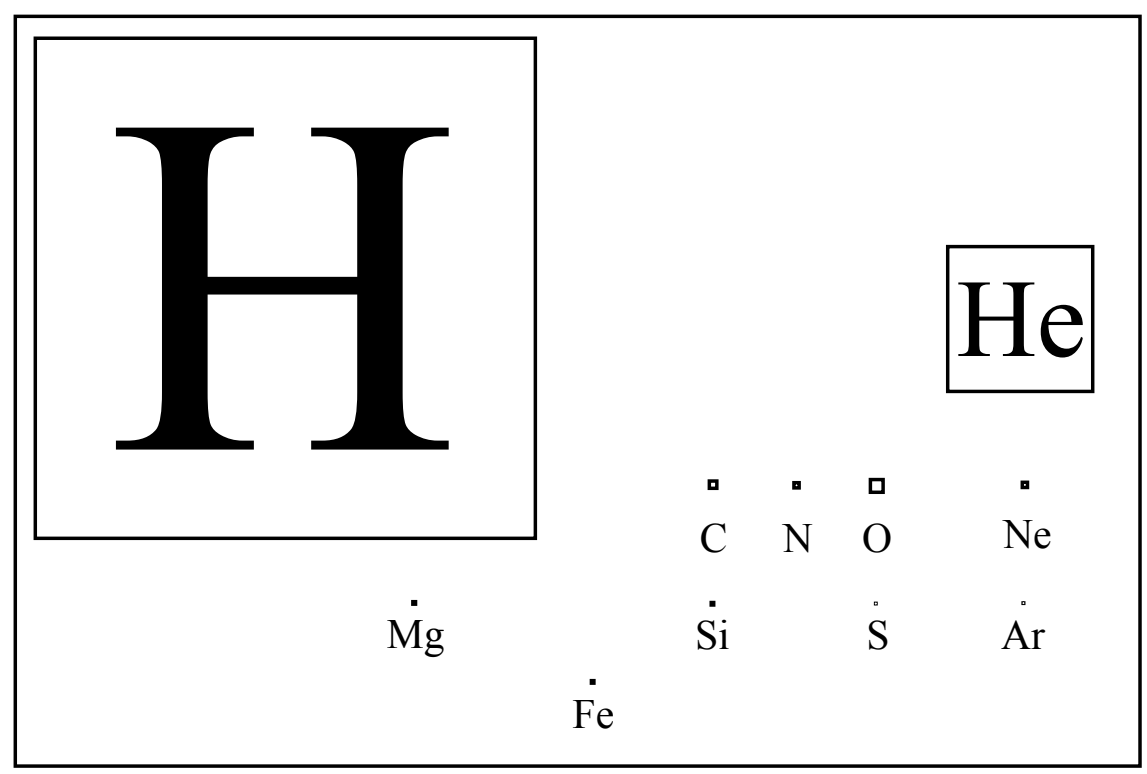

Figure 1. The Astronomer's Periodic Table, in which the area of each element's box is proportional to its cosmic abundance.

higher electron fractions than dense clouds, and these conditions do not seem particularly kind to an active ion-molecule chemistry. For additional background on the physical and chemical conditions in diffuse clouds, the reader is referred to an upcoming Annual Review article (Snow \& McCall 2006).

\section{2. $\mathbf{H}_{3}^{+}$in Diffuse Clouds}

As Figure 1 illustrates, about 90\% of all nuclei in the universe are hydrogen nuclei, and consequently hydrogenic species are very important in astrochemistry. In fact, most of the chemistry in the interstellar medium begins when $\mathrm{H}_{2}$ is ionized by cosmic-ray impact to form $\mathrm{H}_{2}^{+}$, which then quickly reacts with another $\mathrm{H}_{2}$ to form $\mathrm{H}_{3}^{+} \cdot \mathrm{H}_{3}^{+}$is the simplest polyatomic molecule, and can be thought of as molecular hydrogen with an extra proton attached, in an equilateral triangle configuration. Once $\mathrm{H}_{3}^{+}$is formed, it can donate its "extra" proton to just about any atom or molecule, thus initiating a chain of ion-neutral reactions that leads to the production of many of the molecules observed by radioastronomers in dense clouds. This scheme of interstellar chemistry was first proposed independently by Herbst \& Klemperer (1973) and Watson (1973).

As the formation rate of $\mathrm{H}_{3}^{+}$is limited by the cosmic-ray ionization of $\mathrm{H}_{2}$, this rate can be expressed as $\zeta n\left(\mathrm{H}_{2}\right)$, where $\zeta$ is the cosmic-ray ionization rate (typically assumed to be $\left.\sim 3 \times 10^{-17} \mathrm{~s}^{-1}\right)$. In dense molecular clouds, the destruction of $\mathrm{H}_{3}^{+}$is dominated by chemical reaction with $\mathrm{CO}$ to form $\mathrm{HCO}^{+}$, with a rate of $k n\left(\mathrm{H}_{3}^{+}\right) n(\mathrm{CO})$, where $k$ is the rate constant for this reaction (measured in the laboratory to be $\sim 2 \times 10^{-9} \mathrm{~cm}^{3} \mathrm{~s}^{-1}$ ). In steady state, the rates of formation and destruction are equal, and one can solve for the number density of $\mathrm{H}_{3}^{+}$:

$$
n\left(\mathrm{H}_{3}^{+}\right)=\frac{\zeta}{k} \frac{n\left(\mathrm{H}_{2}\right)}{n(\mathrm{CO})} \sim 10^{-4} \mathrm{~cm}^{-3} \quad \text { [dense clouds] }
$$


This quantity is essentially constant through a dense cloud, because $\zeta / k$ is a constant and because the ratio $n\left(\mathrm{H}_{2}\right) / n(\mathrm{CO}) \sim 6700$ is approximately constant (since nearly all $\mathrm{H}$ is in the form of $\mathrm{H}_{2}$ and nearly all $\mathrm{C}$ is in the form of $\left.\mathrm{CO}\right)$. The constancy of $n\left(\mathrm{H}_{3}^{+}\right)$ makes $\mathrm{H}_{3}^{+}$a particularly powerful probe of dense clouds: if one observes the column density $N\left(\mathrm{H}_{3}^{+}\right)$spectroscopically, one can easily infer the column length $L \sim N / n$ and the average hydrogen number density $\left\langle n\left(\mathrm{H}_{2}\right)\right\rangle \sim N\left(\mathrm{H}_{2}\right) / L$.

In diffuse clouds, the formation of $\mathrm{H}_{3}^{+}$is by the same mechanism, but the destruction is dominated by dissociative recombination with the abundant electrons generated by photoionization of atomic carbon. The rate expression for the destruction is then $k_{e} n\left(\mathrm{H}_{3}^{+}\right) n(e)$, where $k_{e}$ is the rate constant for the dissociative recombination. In steady state, the number density of $\mathrm{H}_{3}^{+}$is then:

$$
n\left(\mathrm{H}_{3}^{+}\right)=\frac{\zeta}{k_{e}} \frac{n\left(\mathrm{H}_{2}\right)}{n(e)} \sim 4 \times 10^{-7} \mathrm{~cm}^{-3} \quad \text { [diffuse clouds] }
$$

The quantity $k_{e}$ has been measured many times in the laboratory over the past few decades, but different experiments have given different results, leaving the value of this key quantity somewhat controversial. It has been suggested that a possible reason for the variations between experiments is varying degrees of internal (rotational and vibrational) excitation of the $\mathrm{H}_{3}^{+}$ions used in the various measurements. Recently, storage ring techniques (which ensure complete vibrational relaxation of the $\mathrm{H}_{3}^{+}$) have been combined with rotationally cold ion sources to measure $k_{e}$ under conditions which most closely approximate the interstellar case. Using a supersonic expansion ion source at CRYRING, McCall et al. (2004) measured $k_{e} \sim 1.3 \times 10^{-6} T^{-0.5} \mathrm{~cm}^{3} \mathrm{~s}^{-1}$, and this result has been confirmed by Kreckel et al. (2005) using a cryogenic ion trap at TSR. Here we have adopted a temperature of $\sim 60 \mathrm{~K}$ as used by Le Petit, Roueff, \& Herbst (2004) for the diffuse cloud sightline towards $\zeta$ Persei, and find $k_{e} \sim 1.7 \times 10^{-7} \mathrm{~cm}^{3} \mathrm{~s}^{-1}$. The ratio $n\left(\mathrm{H}_{2}\right) / n(e) \sim 2400$ is approximately constant in diffuse clouds, where roughly half of the hydrogen is in molecular form and nearly all of the carbon is ionized to produce electrons.

Once again, we find a constant number density of $\mathrm{H}_{3}^{+}$(just as in the case of dense clouds), but here the value is considerably lower. Given this low number density, one might expect not to observe $\mathrm{H}_{3}^{+}$in diffuse clouds, but in fact it has been seen in many diffuse cloud sightlines (McCall et al. 1998, 2002, 2003a; Geballe et al. 1999) with column densities of $\sim 10^{14} \mathrm{~cm}^{-2}$, comparable to those in dense clouds. If we take the case of $\zeta$ Persei as an example, the column density $N\left(\mathrm{H}_{3}^{+}\right)=8 \times 10^{13} \mathrm{~cm}^{-2}$, so $L=N / n \sim$ $2 \times 10^{20} \mathrm{~cm} \sim 67 \mathrm{pc}$. Given the known total column density of hydrogen $N_{\mathrm{H}} \equiv N(\mathrm{H})+$ $2 N\left(\mathrm{H}_{2}\right) \sim 1.6 \times 10^{21} \mathrm{~cm}^{-2}$, we would infer an average number density $\left\langle n_{\mathrm{H}}\right\rangle \sim 8 \mathrm{~cm}^{-3}$. However, this is considerably lower than the density inferred from observations of other molecules, which give densities around $150-500 \mathrm{~cm}^{-3}$.

This represents quite a discrepancy, even by astronomical standards! Essentially, the predicted value of $n\left(\mathrm{H}_{3}^{+}\right)$yields a path length that is too long (or equivalently a density that is too low) by a factor of $\sim 20-60$. But equation (2.2) above is quite simple and really contains only three parameters: the cosmic-ray ionization rate $\zeta$, the dissociative recombination rate constant $k_{e}$, and the electron fraction $n(e) / n\left(\mathrm{H}_{2}\right)$. In the case of $\zeta$ Persei, the electron fraction can be directly inferred from spectroscopic observations of the column densities of $\mathrm{H}_{2}$ and $\mathrm{C}^{+}$, and is consistent with complete ionization of carbon as suggested above. This leaves only $\zeta$ and $k_{e}$ as possible suspects for the "overabundant" $\mathrm{H}_{3}^{+}$. It is, of course, conceivable that both storage ring measurements of $k_{e}$ are incorrect and that the "real" value of $k_{e}$ is a factor of 20-60 lower than adopted here. But it is somewhat difficult to accept that both experiments could be wrong, especially since their 
value of $k_{e}$ has found recent support from theoretical calculations (Kokoouline \& Greene 2003).

It seems more likely that the cosmic-ray ionization rate $\zeta$ is a factor of $\sim 20-60$ times higher than expected, or roughly $\sim 1.2 \times 10^{-15} \mathrm{~s}^{-1}$ rather than $\sim 3 \times 10^{-17} \mathrm{~s}^{-1}$. Such a high ionization rate was originally envisaged by Spitzer \& Tomasko (1968), but was later abandoned when observations suggested the value $\sim 3 \times 10^{-17} \mathrm{~s}^{-1}$ (see the discussion by Dalgarno). The concept of a high value of $\zeta$ in diffuse clouds has recently regained some support, however (Liszt 2003; Le Petit, Roueff, \& Herbst 2004; Padoan \& Scalo 2005). It now seems that spectroscopic observations of $\mathrm{H}_{3}^{+}$in diffuse clouds may serve as a valuable probe of the cosmic-ray ionization rate, which is otherwise difficult to constrain observationally.

\section{Carbon Chains in Diffuse Clouds}

The shortest carbon chain, $\mathrm{C}_{2}$, was discovered in diffuse clouds by Souza \& Lutz (1977) using the $A-X 1-0$ band near $1.015 \mu \mathrm{m}$ in the sightline towards Cygnus OB2 12. Following this initial detection, many spectra of $\mathrm{C}_{2}$ in diffuse clouds have been obtained, primarily using the $2-0$ band near $8750 \AA$, which is more easily accessible with CCD-based spectrographs. As described by van Dishoeck \& Black (1982), the observed rotational excitation of $\mathrm{C}_{2}$ can be used as a probe of both the density and the kinetic temperature of the absorbing gas. An online "calculator" for extracting density and temperature from observed $\mathrm{C}_{2}$ spectra is available at dibdata.org.

It was not until 2001 that the next longest carbon chain, $\mathrm{C}_{3}$, was detected in diffuse clouds, by Maier et al. (2001) using the $A-X \quad 0-0$ band near $4051 \AA$. These authors detected $\mathrm{C}_{3}$ towards $\zeta$ Oph, $\zeta$ Per, and 20 Aql, with rotational resolution. Shortly thereafter, Roueff et al. (2002) detected $\mathrm{C}_{3}$ towards HD 210121 and developed a full excitation model (like in the case of $\mathrm{C}_{2}$ ) to infer the temperature and number density. Unfortunately, the details of this model have yet to be published, and so it is not available to analyze the many spectra of $\mathrm{C}_{3}$ that have been subsequently obtained. In 2003, Oka et al. (2003) published a number of $\mathrm{C}_{3}$ spectra resulting from the Apache Point Observatory diffuse interstellar band survey (described below). Although these spectra had lower resolution and were therefore not rotationally resolved, they demonstrated the widespread occurrence of $\mathrm{C}_{3}$ and showed a rough correlation between the $\mathrm{C}_{2}$ and $\mathrm{C}_{3}$ column densities.

To follow up on the $\mathrm{C}_{3}$ results of the Oka survey, Máté Ádámkovics, Geoff Blake, and I began a campaign to obtain higher resolution spectra of Oka's sightlines using the Lick and Keck observatories. From this campaign we obtained rotationally resolved spectra of $\mathrm{C}_{3}$ in 10 sightlines (Ádámkovics, Blake, \& McCall 2003). In the course of this survey, we noted that the wavelength of the $R(0)$ line did not seem to match the laboratory values reported by Gausset et al. (1965); a subsequent experiment using cavity ringdown spectroscopy on a supersonically expanding plasma (which produces rotationally cold $\mathrm{C}_{3}$ ) confirmed that the original laboratory value was in error (McCall et al. 2003b).

Having been the first to detect $\mathrm{C}_{3}$, Maier and co-workers turned their attention to the next longest carbon chains $\mathrm{C}_{4}$ and $\mathrm{C}_{5}$, whose laboratory spectra in the visible region were already known. They obtained very high signal-to-noise ratio spectra towards $\zeta$ Oph, but were not able to detect either molecule (Maier, Walker, \& Bohlender 2004). Although $\zeta$ Oph is very bright $(V=2.56)$, and therefore one can obtain high $\mathrm{S} / \mathrm{N}$ in a short time, it does not have particularly high $\mathrm{C}_{2}$ and $\mathrm{C}_{3}$ column densities. A sightline such as HD 204827, which has very high column densities of both $\mathrm{C}_{2}$ and $\mathrm{C}_{3}$, seemed a better bet, although it is much fainter $(V=7.94)$. Recently, Máté Ádámkovics, Geoff Blake, and I spent two nights at Keck to obtain a very high S/N spectra of HD 204827-sadly, 
our effort did not yield a detection of either $\mathrm{C}_{4}$ or $\mathrm{C}_{5}$ (Ádámkovics, Blake, \& McCall 2006). Our limit on the $\mathrm{C}_{4}$ column density is roughly a third of the $\mathrm{C}_{3}$ column density, and that on the $\mathrm{C}_{5}$ column density is roughly one-sixteenth of $\mathrm{C}_{3}$.

The lack of observed $\mathrm{C}_{4}$ and $\mathrm{C}_{5}$ is somewhat surprising, and must be telling us something about the chemistry of these clouds. To our knowledge, the only chemical model of diffuse clouds that includes $\mathrm{C}_{4}$ and $\mathrm{C}_{5}$ is that of Roueff et al. (2002). This model targets the sightline towards $\mathrm{HD} 210121$, and suggests that at visual extinctions greater than about 1.3 , the $\mathrm{C}_{4}$ column density exceeds that of $\mathrm{C}_{3}$. At $A_{V} \sim 1.8, \mathrm{C}_{5}$ overtakes $\mathrm{C}_{3}$. If we näively extrapolate the figure in their paper to $A_{V}=2.6$ (for HD 204827), we find the $\mathrm{C}_{4}$ should have twice the column density of $\mathrm{C}_{3}$ ! Clearly there is a conflict between the observations and the model, and we encourage chemical modellers to work on this very interesting sightline towards HD 204827.

\section{The Diffuse Interstellar Bands}

The diffuse interstellar bands (DIBs) are an enigmatic set of at least 200 absorption lines observed in the visible spectra of nearly all stars whose light is filtered through interstellar gas and dust. The first of the DIBs, $\lambda 5780$ and $\lambda 5797$ were observed by Mary Lea Heger at Lick Observatory in 1919. The DIBs seem, for the most part, to be associated with diffuse atomic clouds; their intensity is roughly correlated with the visual extinction, but at high visual extinctions their intensity tends to level off. The DIBs have been reviewed by Herbig (1995) and are also discussed by Snow \& McCall (2006).

For a long time, the rough correlation with the visual extinction led researchers to favor solid state carriers for the DIBs. However, several characteristics of the DIBs argue strongly against dust as the carrier. These characteristics include the constancy of the central wavelengths, the lack of any associated emission features, and the fine structure that has been observed at high resolution in some DIBs (e.g., Sarre et al. 1995). Today, it is generally accepted that the DIBs are caused by large polyatomic molecules in the gas phase. Most likely the DIB carriers are carbon-based, and constitute a vast reservoir of organic material in the interstellar medium. The problem of the DIBs has been called the greatest unsolved mystery in all of spectroscopy. The eventual identification of the molecules causing the DIBs will more than double the known inventory of interstellar molecules, and will provide a powerful multidimensional probe of the chemical and physical conditions in interstellar clouds.

In an effort to compile a high-quality set of DIB spectra that can be used both for studying the DIBs themselves as well as for comparison with laboratory spectra of candidate carriers, a large-scale survey has recently been conducted at the Apache Point Observatory in New Mexico. This survey has utilized the ARCES (Astrophysical Research Consortium Echelle Spectrograph), which offers a resolving power $\lambda / \Delta \lambda \sim 37,500$ and complete spectral coverage from 3600-10,200 A. Our main campaign involved observations on 119 nights from January 1999 through January 2003, and yielded spectra with signal-to-noise ratios (at $5780 \AA$ ) greater than 500 for 160 stars (115 of which are reddened). The measurements and analysis of the DIBs in these spectra are still very much underway, but I'd like to discuss some of our early results.

As mentioned above, most of the DIBs appear to be associated with diffuse atomic clouds. For example, Herbig (1993) found a better correlation between the strength of the $\lambda 5780$ band with the column density of atomic hydrogen than with that of molecular hydrogen. Preliminary results from the APO survey are shown in Figure 2 and confirm Herbig's findings with much greater clarity (York et al. 2006). The fact that most DIBs 

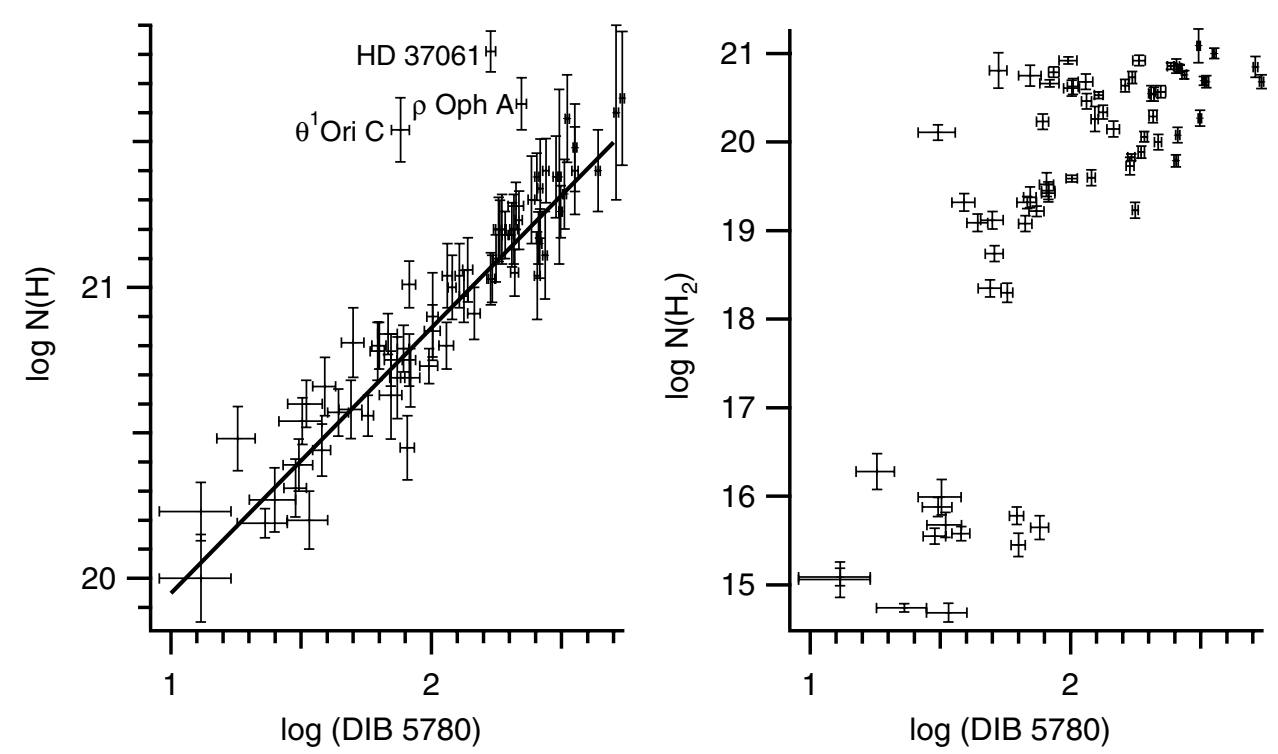

Figure 2. The good correlation (left) between $\lambda 5780$ and the column density of H I, compared with the bad correlation (right) between $\lambda 5780$ and $\mathrm{H}_{2}$. For $\mathrm{H} \mathrm{I}$ and $\lambda 5780$, the correlation coefficient is $r=0.94$, excluding the three outliers (which have unusual radiation fields).

are found in gas where the hydrogen molecular fraction is low represents an important chemical constraint on the types of molecules responsible for the DIBs.

However, not all DIBs seem to follow this behavior. The APO survey has yielded the identification of a set of DIBs (now called the " $\mathrm{C}_{2}$ DIBs") whose intensities show some correlation with the column density of $\mathrm{C}_{2}$ (Thorburn et al. 2003). Since $\mathrm{C}_{2}$ is known to exist in the denser regions of diffuse clouds, which are probably more molecular, these $\mathrm{C}_{2}$ DIBs seem to form a distinct class from the classical DIBs like $\lambda 5780$, which correlate with atomic hydrogen. Further evidence for this separation of the DIBs into two classes has been provided by observations of the peculiar line of sight towards HD 62542, which appears to have been stripped of its outer, more diffuse layers by a shock. Snow et al. (2002) failed to detect DIBs in this sightline, but a more recent higher $\mathrm{S} / \mathrm{N}$ study by Ádámkovics, Blake, \& McCall (2005) detected weak DIBs. Most interestingly, the $\mathrm{C}_{2}$ DIBs were clearly observed, while many of the classical DIBs could not be detected at all. This strongly suggests that the $\mathrm{C}_{2}$ DIBs are abundant in the "cores" of diffuse clouds (that is, in diffuse molecular clouds, using the classification scheme of Snow \& McCall 2006) whereas the classical DIBs are abundant in the "envelopes" of diffuse clouds (that is, in diffuse atomic clouds).

As part of our APO survey, we have been searching for pairs of DIBs whose intensities are well-correlated in different sightlines. The motivation for this search is that most molecules show more than one vibronic band in their electronic spectra, and that (in the absence of low-lying splittings in the electronic ground state) the relative intensities of these vibronic transitions are governed only by the Franck-Condon factors, not by environmental conditions. Therefore, a set of DIBs that are "perfectly" correlated could be expected to represent the electronic spectrum of a single molecular carrier, and the spacings among those DIBs could give insight into the nature of that carrier. To date, we have only searched for correlations among the strongest DIBs, and have found relatively 


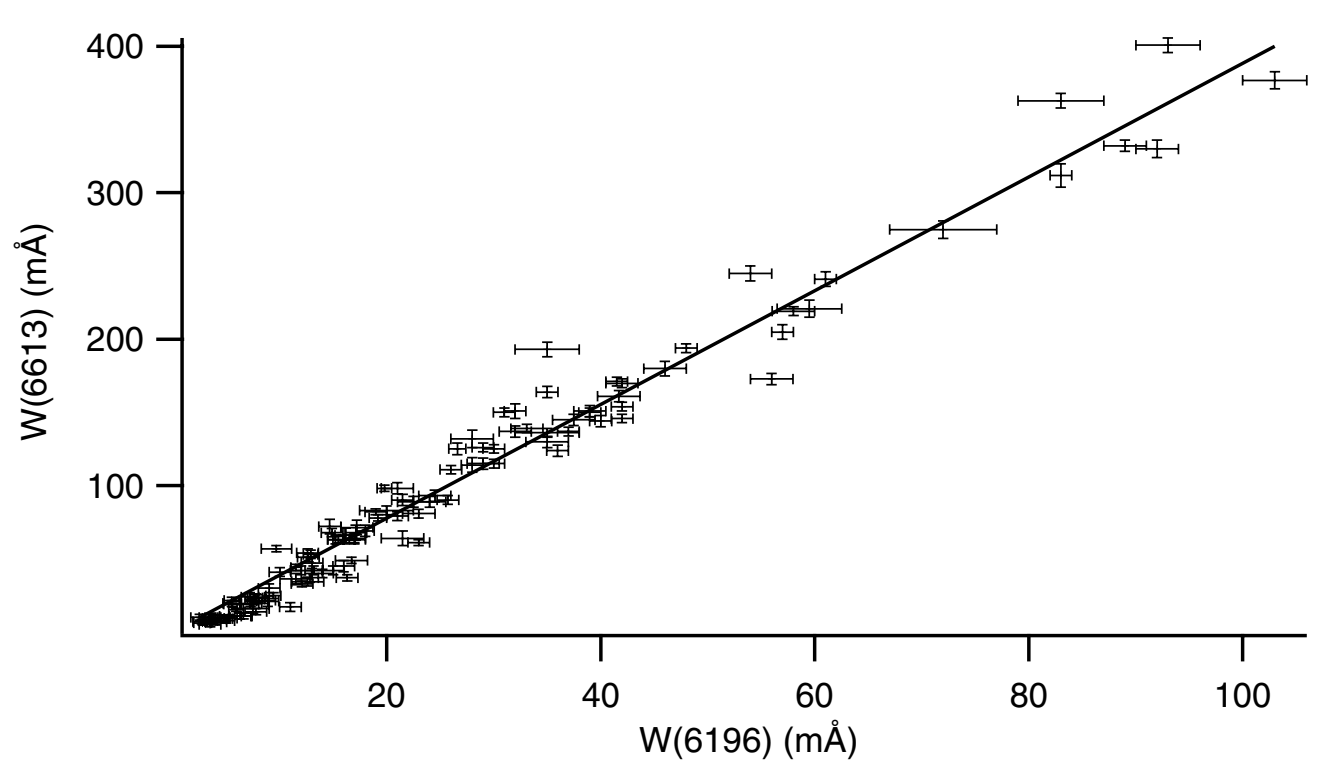

Figure 3. The remarkably good correlation between $\lambda 6196$ and $\lambda 6613$. The correlation coefficient is $r=0.986$.

few correlations which might be considered "perfect." The best correlation we have found so far is that between $\lambda 6196$ and $\lambda 6613$, as shown in Figure 3 . The correlation coefficient for this pair of DIBs is $r=0.986$ in our sample, which is not unity but is pretty close. It appears that the deviation from a perfect straight line is larger than would be expected from our estimated uncertainties, which suggests either that we have underestimated our errors, or that these two DIBs are not due to the same molecule but instead to two very closely related molecules. However, we consider the quality of this correlation to be quite striking, especially by astronomical standards.

\section{Conclusions}

Just ten years ago, the conventional wisdom was that diffuse interstellar clouds were relatively uninteresting chemically, consisting mainly of atoms and a few diatomic molecules. In the past decade, infrared and optical spectroscopy have demonstrated the ubiquity of $\mathrm{H}_{3}^{+}$and $\mathrm{C}_{3}$ in diffuse clouds, and observations at radio wavelengths have detected many other polyatomics (Liszt, this volume). It is becoming increasingly clear that diffuse clouds are chemically rich, and that our understanding of their chemistry is still very immature. Perhaps our greatest reminder of this fact is the existence of the diffuse interstellar bands and the fact that the identities of their carriers still elude us over 85 years since their first observation. We can expect the next decade to be an incredibly exciting one for chemical studies of diffuse clouds, in terms of both observations and modelling. With increasing sensitivity of both optical and infrared spectroscopy, many new molecules may be detected. With increasing sophistication of chemical models of diffuse clouds, we may finally understand some of the puzzling aspects of diffuse cloud chemistry. And, with a high-quality database of DIB spectra, coupled with ever more sophisticated laboratory experiments, we may even identify some of the carriers of the DIBs! 


\section{Acknowledgments}

It is a pleasure to acknowledge the contributions of my collaborators. The $\mathrm{H}_{3}^{+}$observations were conducted with T. R. Geballe and T. Oka. The storage-ring measurements of the $\mathrm{H}_{3}^{+}$dissociative recombination rate involved a large team headed up by M. Larsson which included A. J. Huneycutt, R. J. Saykally, N. Djuric, G. H. Dunn, J. Semaniak, O. Novotny, A. Paal, F. Österdahl, A. Al-Khalili, A. Ehlerding, F. Hellberg, S. Kalhori, A. Neau, and R. Thomas. The observations of $\mathrm{C}_{3}, \mathrm{C}_{4}$, and $\mathrm{C}_{5}$ were conducted with $\mathrm{M}$. Ádámkovics and G. A. Blake. The APO DIB survey has included contributions from S. D. Friedman, L. M. Hobbs, T. Oka, B. L. Rachford, T. P. Snow, P. Sonnentrucker, J. A. Thorburn, D. E. Welty, and D. G. York.

I also wish acknowledge financial support from the NASA Laboratory Astrophysics program, a National Science Foundation CAREER award, a Dreyfus New Faculty award, an American Chemical Society Petroleum Research Fund grant, and the University of Illinois.

\section{References}

Ádámkovics, M., Blake, G.A., \& McCall, B.J. 2003, Ap. J. 595, 235

Ádámkovics, M., Blake, G.A., \& McCall, B.J. 2005, Ap. J. 625, 857

Ádámkovics, M., Blake, G.A., \& McCall, B.J. 2006, in preparation

Gausset, L., Herzberg, G., Lagervist, A., \& Rosen, B. 1965, Ap. J. 142, 45

Geballe, T.R., McCall, B.J., Hinkle, H.K., \& Oka, T. 1999, Ap. J. 510, 251

Herbig, G.H. 1993, Ap. J. 407, 142

Herbig, G.H. 1995, ARA\&A 33, 19

Herbst, E. \& Klemperer, W. 1973, Ap. J. 185, 505

Kokoouline, V. \& Greene, C.H. 2003, Phys. Rev. A, 68, 012703

Kreckel, H. et al. 2005, Phys. Rev. Lett. 95, 263201

Le Petit, F., Roueff, E., \& Herbst, E. 2004, A\& A 417, 993

Liszt, H. 2003, A $\mathcal{E} A$ 398, 621

Maier, J.P, Lakin, N.M, Walker, G.A.H., \& Bohlender, D.A. 2001, Ap. J. 553, 267

Maier, J.P, Walker, G.A.H., \& Bohlender, D.A. 2004, Ap. J. 602, 286

McCall, B.J., Geballe, T.R., Hinkle, K.H., \& Oka, T. 1998, Science 279, 1910

McCall, B.J. et al. 2002, Ap. J. 567, 391

McCall, B.J. et al. 2003a, Nature 422, 500

McCall, B.J., Casaes, R.N, Ádámkovics, M., \& Saykally, R.J. 2003b, Chem. Phys. Lett. 374, 583

McCall, B.J. et al. 2004, Phys. Rev. A 70, 052716

Oka, T. et al. 2003, Ap. J. 582, 823

Padoan, P. \& Scalo, J. 2005, Ap. J. 624, L97

Roueff, E., Felenbok, P., Black, J.H., \& Gry, C. 2002, A\&\&A 384, 629

Sarre, P.J. et al. 1995, MNRAS 277, L41

Snow, T.P. et al. 2002, Ap. J. 573, 670

Snow, T.P. \& McCall, B.J. 2006, $A R A \& A$, in preparation

Souza, S.P. \& Lutz, B.L. 1977, Ap. J. 216, L49

Spitzer, L. \& Tomasko, M.G. 1968, Ap. J. 152, 971

Thorburn, J. et al. 2003, Ap. J. 584, 339

van Dishoeck, E.F. \& Black, J.H. 1982, Ap. J. 258, 533

Watson, W.D. 1973, Ap. J. 183, L17

York, D.G. 2006, in preparation 


\section{Discussion}

KreŁowski: (1) Despite the very good correlation between the 6196 and 6614 DIBs, (found by Moutou et al. 1999, A\&SA 351, 680) their profiles do not change in unison (Galazutdinov et al. 2002, A\&SA 384, 215). (2) In the case of $\mathrm{C}_{5}$, nature proves once again its complexity. While the $5109 \AA$ feature, predicted by the laboratory experiments is apparently absent in spectra of reddened stars, the second feature, $4975 \AA$, has a counterpart, most likely a weak DIB (Galazutdinov et al. 2002, A $\mathscr{G} A$ 395, 223). (3) The " $\mathrm{C}_{2}$ DIBs" reported in your lecture correlate in our sample to a various degree with the $\mathrm{C}_{2}$ column density. However, the strength ratios of these DIBs are variable. What do they have in common?

MCCALL: As discussed in detail by Thorburn et al. 2003 (Ap. J. 584, 339), the "C 2 DIBs" are a set of DIBs whose equivalent widths (normalized to $\lambda 6196$ ) are well correlated with $N\left(\mathrm{C}_{2}\right) / E(B-V)$. We do not suggest that they belong to the same molecule, only that (unlike other DIBs) they are correlated with $\mathrm{C}_{2}$.

Galazutdinov: While relating the abundances of $\mathrm{C}_{2}$ and $\mathrm{C}_{3}$ molecules, have you applied the data from the highest resolution study of $\mathrm{C}_{3}$ published until now (Galazutdinov et al. 2002, A\&A 395, 969)?

MCCALL: In our comparison of $\mathrm{C}_{2}$ and $\mathrm{C}_{3}$ column densities, we have utilized the equivalent widths for the three sources (HD 148184, 149757, and 152236), for which you reported $\mathrm{C}_{3}$ detections. However, we had to recompute the column densities as the values in your paper were incorrect (too low) by nearly an order of magnitude.

SARRE: Concerning the " $\mathrm{C}_{2}$ " diffuse bands, have you investigated if there is any link between the $\mathrm{C}_{2}$ rotational temperatures and the diffuse band strengths?

MCCALL: No, we have not looked into that, but it is a very interesting suggestion!

RouefF: Extrapolation of $\mathrm{C}_{4}$ and $\mathrm{C}_{5}$ modeled column densities is not acceptable. Can you make precise the errorbars in the determination of the column density of $\mathrm{H}_{3}^{+}$?

MCCALL: I hope that my unacceptable extrapolation will serve as encouragement for new chemical models of this sightline! I do not have the calculated error bars off the top of my head, but we are preparing a manuscript with such observational details. I would estimate that the $\mathrm{H}_{3}^{+}$column density towards $\zeta$ Per is good to at least a factor of two. 


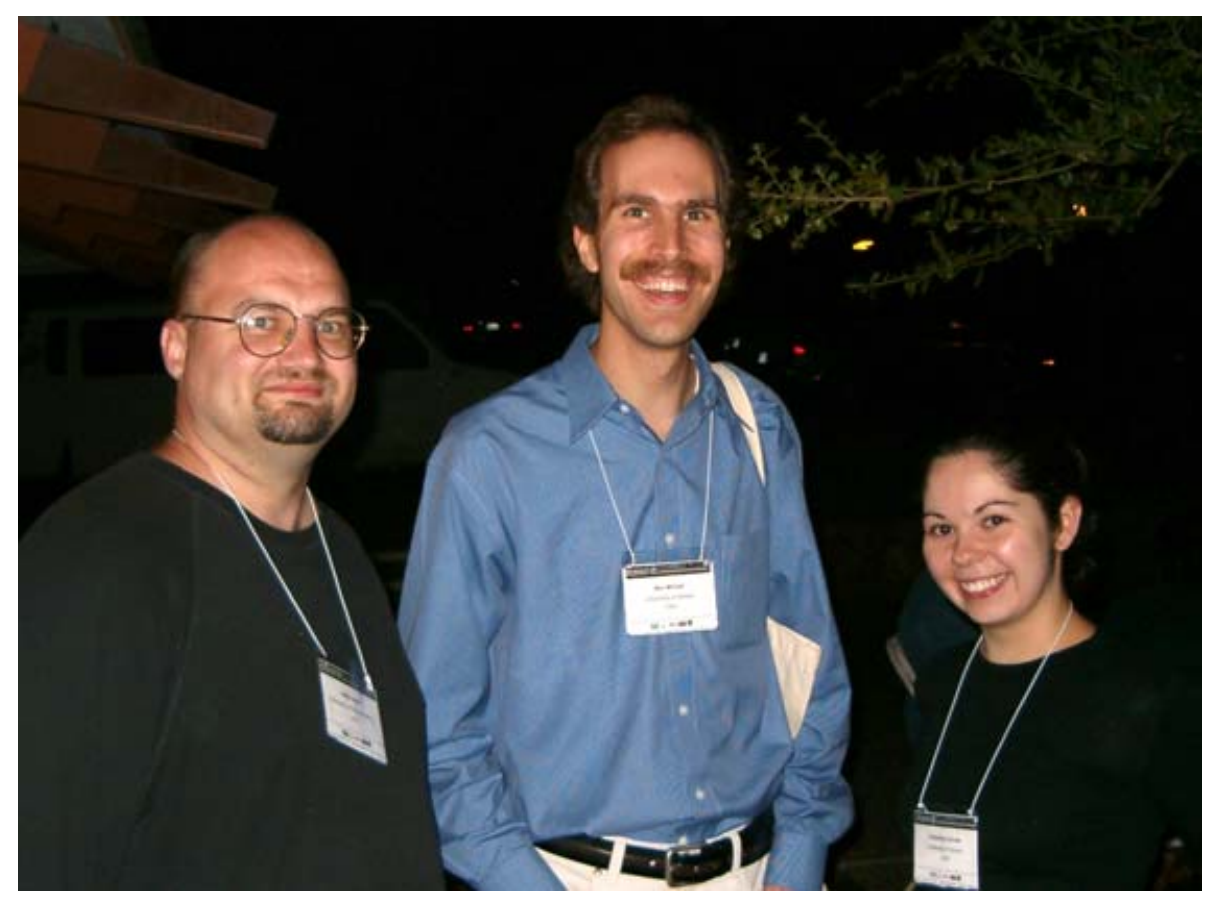

Photo: E. Herbst 